\title{
Calcium Plus Vitamin D Supplementation Is Not Associated with a Reduced Breast Cancer Risk
}

Taking calcium and vitamin D supplements does not reduce breast cancer incidence in postmenopausal women, according to data from a randomized, doubled-blind, placebo-controlled trial published online November 11 in the fournal of the National Cancer Institute.

Several observational and preclinical studies have suggested that vitamin D supplements may reduce breast cancer risk, but results have been inconsistent.

To test this possibility, researchers evaluated breast cancer incidence as a secondary endpoint in the Women's Health Initiative study in 36,282 postmenopausal women who were randomly assigned to take 1,000 $\mathrm{mg}$ of calcium plus $400 \mathrm{IU}$ of vitamin D daily or to daily placebo. (The primary endpoint of the study was effect of the supplements on hip fracture.) Baseline 25-hydroxyvitamin D levels were determined in all 1,067 breast cancer cases and 1,067 matched control subjects.

In the current analysis, Rowan Chlebowski, M.D., Ph.D., of the Los Angeles Biomedical Research Institute at HarborUniversity of California Los Angeles Medical Center and colleagues found that the incidence of invasive breast cancer was similar in the supplement and placebo groups, with 528 and 546 cases, respectively. Additionally, 25-hydroxyvitamin D levels were only modestly associated with dietary and supplement vitamin D intake and were not associated with breast cancer risk in a nested case-control analysis.

These findings call into question recommendations for evaluation of higher vitamin D dosage in future studies, according to the authors. Furthermore, as 25-hydroxyvitamin D levels were strongly associated with leanness and high physical activity, which both influence breast cancer risk, prior observational studies relating vitamin D levels to breast cancer risk may have been influenced by these factors. "The main findings do not support a causal relationship between calcium and vitamin D supplement use and reduced breast cancer incidence" the authors conclude.

In an accompanying editorial, Corey Speers and Powel Brown, M.D., Ph.D., of Baylor College of Medicine in Houston applaud the authors' efforts to test the question in such a rigorous manner. Although the editorialists agree that these data do not support an association between calcium and vitamin D supplementation and breast cancer incidence, they argue that further investigation is still needed to adequately answer the question, including looking for genetic influences on vitamin $\mathrm{D}$ serum levels, testing supplements in premenopausal women, and following study participants for a longer follow-up period.

"Because preclinical, epidemiological, and clinical trial results of vitamin D supplementation are conflicting, additional studies will be needed to determine whether vitamin D plus calcium will prevent breast cancer. However, this article by Chlebowski [and colleagues] offers an important first step in addressing this issue," the editorialists write. "The potential health benefits of vitamin D and calcium may yet still have a bright future."

\section{Contact:}

- Article: Laura Mecoy, lmecoy@issuesmanagement.com, (310) 546-5860 (office) and (310) 529-7717 (Mobile)

- Editorial: Megan Holtze, mhotze@bcm.tmc.edu, (713) 798-4564 


\section{Citation:}

- Article: Chlebowski RT, Johnson KC, Kooperberg C, et al. Calcium Plus Vitamin D Supplementation and the Risk of Breast Cancer. J Natl Cancer Inst 2008;100:1581-1591

- Editorial: Speers C, Brown P. Breast Cancer Prevention Using Calcium and Vitamin D: A Bright Future? J Natl Cancer Inst 2008;100:1562-1564 